\title{
Terapia sostitutiva corticosteroidea alla luce delle nuove proposte farmacologiche (Plenadren ${ }^{\circledR}$ )
}

\author{
Vincenzo Cappiello ${ }^{1}$ Jacopo Giannelli ${ }^{1} \cdot$ Roberta Giordano $^{2}$
}

Accettato: 26 settembre 2020 / Pubblicato online: 13 maggio 2021

(c) The Author(s) 2021

Sommario La terapia sostitutiva corticosteroidea è indispensabile per la sopravvivenza dei pazienti con insufficienza surrenalica. Per oltre cinquant'anni sono stati impiegati steroidi a breve emivita e solo negli ultimi vent'anni sono state proposte nuove formulazioni derivate dall'idrocortisone, nate con l'obiettivo di migliorare gli effetti delle terapie convenzionali. In particolare, è stata prodotta una formulazione di idrocortisone a rilascio modificato in due fasi (DR-HC, Plenadren ${ }^{\circledR}$ ). In questa rassegna si descriveranno le caratteristiche e gli effetti di tale formulazione.

Parole chiave Insufficienza surrenalica - Terapia sostitutiva $\cdot$ Idrocortisone $\cdot$ QoL $\cdot$ Morbilità $\cdot$ Mortalità

\section{Introduzione}

La terapia sostitutiva corticosteroidea è indispensabile per la sopravvivenza dei pazienti con insufficienza surrenalica.

L'obiettivo della terapia sostitutiva è quello di riprodurre il normale profilo circadiano della secrezione surrenalica di

Proposto da Daniele Santi.

Informazioni Supplementari La versione online contiene materiale supplementare disponibile su

https://doi.org/10.1007/s40619-021-00860-1.

$\varangle$ R. Giordano

roberta.giordano@unito.it

1 Dip. Scienze Mediche, SCDU Endocrinologia, Diabetologia e Metabolismo, AOU Città della Salute e della Scienza di Torino, Torino, Italia

2 Dip. Scienze Cliniche Biologiche, Università di Torino, SCDU Endocrinologia, Diabetologia e Metabolismo, AOU Città della Salute e della Scienza di Torino, Torino, Italia cortisolo, al fine di ridurre i segni e i sintomi della malattia ed evitare condizioni di sotto- e sovradosaggio.

Per oltre cinquant' anni sono state utilizzate formulazioni di glucocorticoidi a rapido rilascio, caratterizzate da una breve emivita e, pertanto, somministrate per via orale in multiple dosi giornaliere (bi- o tri-giornaliere), con dosi totali variabili da 15 a $25 \mathrm{mg}$ al dì per l'idrocortisone e $20-35 \mathrm{mg}$ al dì per il cortone acetato, come raccomandato sia dal Consensus Statement del 2013 [1] che dalle Linee Guida dell'Endocrine Society del 2016 [2].

Tali schemi terapeutici presentano, tuttavia, alcune limitazioni legate all'incapacità di ripristinare il ritmo circadiano della secrezione endogena del cortisolo, con picchi di cortisolemia dopo l'assunzione orale e livelli di cortisolemia indosabili prima della terapia stessa. Pertanto, con la terapia convenzionale i pazienti con insufficienza surrenalica presentano livelli di mortalità più alti rispetto alla popolazione generale, almeno secondo alcuni studi [3], e un aumentato rischio di co-morbidità, soprattutto di patologie cardiovascolari, obesità e alterazioni metaboliche [4, 5], oltre che disturbi del sonno, aumentato rischio di osteoporosi e di fratture [4].

Negli ultimi vent'anni sono state proposte nuove formulazioni derivate dall'idrocortisone, nate con l'obiettivo di migliorare gli effetti delle terapie convenzionali, tra cui una nuova formulazione di idrocortisone a doppio rilascio (DR-HC, Plenadren ${ }^{\circledR}$ ), ideato per essere assunto in monodose giornaliera, con l'obiettivo pertanto di aumentare la compliance del paziente e per migliorare le caratteristiche farmacocinetiche e farmacodinamiche dell'idrocortisone stesso [6].

In questa rassegna si descriveranno le caratteristiche farmacologiche del Plenadren ${ }^{\circledR}$ e si riporteranno gli effetti di tale farmaco su differenti parametri, incluso il profilo di sicurezza. 


\section{Farmacocinetica}

Il DR-HC (inizialmente con il nome commerciale di "Duocort", successivamente di Plenadren ${ }^{\circledR}$ ) è stato prodotto in Svezia nel 2009 [6] come una formulazione costituita da una parte interna (core), contenente idrocortisone a rilascio prolungato, e da un rivestimento esterno, contenente idrocortisone a rilascio immediato. La porzione esterna viene resa immediatamente disponibile e assorbita rapidamente grazie alla sua elevata permeabilità intestinale. La parte interna rimanente viene rilasciata a un ritmo più lento nell'intestino tenue e crasso. Il Plenadren ${ }^{\circledR}$ è disponibile in compresse da 5 e $20 \mathrm{mg}$.

La farmacocinetica delle compresse di DR-HC è stata studiata per la prima volta da Johannsson e collaboratori nel 2009 , in uno studio di fase 1 , randomizzato, controllato, a 2 vie, doppio-cieco, condotto in 16 soggetti adulti sani (9 maschi e 7 femmine, $18-65$ anni, BMI $18-27 \mathrm{~kg} / \mathrm{m}^{2}$ ), in cui la secrezione endogena di cortisolo era bloccata previo trattamento con betametasone [6]. Sono state valutate le caratteristiche farmacocinetiche della formulazione assunta sia a digiuno, per entrambe le dosi, sia dopo il pasto per la dose di $20 \mathrm{mg}$ [6].

Gli autori hanno dimostrato che quando il DR-HC viene assunto a digiuno l'assorbimento è rapido, essendo necessari solo 17-20 minuti per il raggiungimento di una cortisolemia pari a $200 \mathrm{nmol} / \mathrm{l}$ per la compressa da $20 \mathrm{mg}$. Inoltre, si è osservato che il picco di cortisolemia raggiunto dal DR-HC è simile a quello dell' $\mathrm{HC}$ convenzionale, impiegando la molecola circa 40-50 minuti per raggiungerlo. Non sono state osservate differenze in termini di Tmax tra le due formulazioni da 5 e $20 \mathrm{mg}$ assunte a digiuno. Gli autori hanno pertanto dimostrato che il DR-HC presenta un rapido assorbimento intestinale con una biodisponibilità superiore al $90 \%$. È stato inoltre osservato che il DR-HC ha provocato un calo più graduale della cortisolemia rispetto all' $\mathrm{HC}$, presentando un'emivita terminale più lunga di circa $1,0-1,5$ ore rispetto all'HC, senza differenze tra le due formulazioni da $5 \mathrm{e}$ $20 \mathrm{mg}$. L'emivita terminale del DR-HC è risultata comunque sufficientemente corta da evitare livelli sovra-fisiologici di cortisolo sia al pomeriggio, osservati con l'HC, che in serata (Fig. 1).

Nello stesso studio sono stati poi valutati gli effetti di una dose di DR-HC da $20 \mathrm{mg}$ assunta 30 minuti dopo una colazione ad alto contenuto calorico, dimostrando come l'assorbimento intestinale del DR-HC risulta ritardato di circa 60 minuti rispetto all'assunzione a digiuno, senza peraltro differenze nel profilo della cortisolemia, che risulta simile a quello registrato dopo somministrazione del DR-HC a digiuno.

Nel 2012, gli stessi autori hanno condotto un secondo studio multicentrico, di fase 2 , aperto, randomizzato, con primo periodo crossover di durata di 12 settimane e un secondo periodo di estensione di durata di 24 settimane, in cinque Cen-

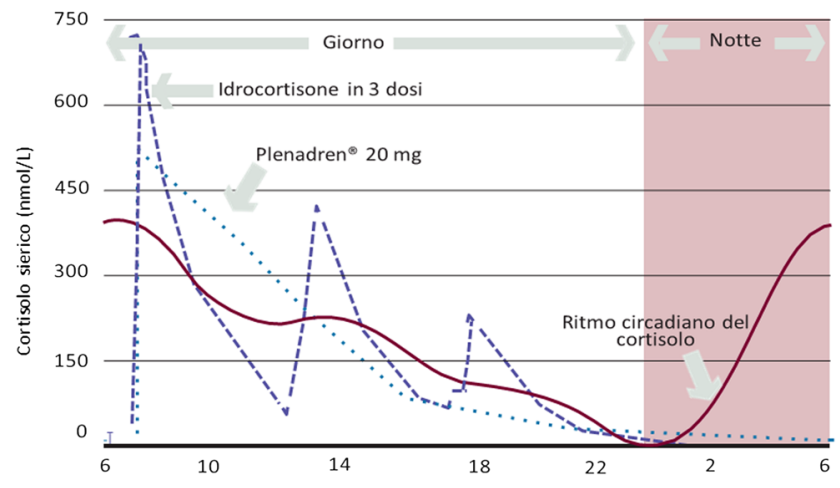

Fig. 1 Livelli di cortisolo sierico dopo somministrazione di tre dosi $(10+5+5 \mathrm{mg})$ di idrocortisone, una singola dose $(20 \mathrm{mg})$ di Plenadren e durante secrezione endogena

tri Ospedalieri Universitari, in 63 pazienti affetti da insufficienza surrenalica primaria [7]. Lo studio ha confrontato la farmacocinetica di DR-HC assunto una volta al giorno con la stessa dose di HC somministrata tre volte al giorno (TID). I risultati di questo studio hanno confermato un profilo di cortisolemia migliore con la formulazione DR-HC rispetto al trattamento TID, con livelli di cortisolemia più elevati durante le prime 4 ore dopo l'assunzione mattutina di DR-HC $\mathrm{e}$, successivamente, livelli più bassi durante il giorno, con una riduzione dell'esposizione al cortisolo nelle 24 ore di circa il $19,4 \%$ rispetto alla TID (Tabella 1 ).

Più recentemente, uno studio randomizzato, aperto, di quattro periodi ha valutato la farmacocinetica del di DR-HC alle dosi di 5, 15 e $20 \mathrm{mg}$ in 31 volontari caucasici giapponesi o non ispanici di età compresa tra 20 e 55 anni, con secrezione endogena di cortisolo soppressa dal desametasone [8]. Gli autori hanno dimostrato che il DR-HC alla dose di $20 \mathrm{mg}$ determinava concentrazioni di cortisolemia più elevate di quelle endogene nelle prime 4 ore dopo l'assunzione, ma le concentrazioni successive erano simili. Inoltre, hanno dimostrato come le concentrazioni di cortisolo e i parametri farmacocinetici sono aumentati con dosi più elevate di DR-HC, ma in modo non proporzionale alla dose somministrata. Infine, gli autori hanno osservato una grande variabilità inter-individuale nei profili di cortisolemia dopo la somministrazione del DR-HC.

Complessivamente, i risultati di questi studi mostrano come il DR-HC presenti un profilo farmacocinetico più favorevole rispetto all'HC (Tabella 1, Fig. 1).

\section{Effetti su parametri antropometrici, metabolici e sul rischio cardiovascolare}

Nel 2012, lo studio di Johannsson e colleghi [7] ha mostrato che dopo 12 settimane di trattamento con DR-HC il peso corporeo e l'emoglobina glicosilata (HbA1C) diminuivano significativamente nell'intera coorte di pazienti affetti da 
Tabella 1 Studi clinici sulle caratteristiche farmacocinetiche del DR-HC. PAI, insufficienza surrenalica primaria; DM, diabete mellito

\begin{tabular}{|c|c|c|c|c|}
\hline Autore & Tipo di studio & Soggetti & durata del trt (dose) & Risultati principali \\
\hline Johannsson et al. [6] & $\begin{array}{l}\text { fase } 1 \text {, randomizzato, } \\
\text { controllato, } 2 \text { vie, } \\
\text { doppio-cieco }\end{array}$ & $\begin{array}{l}16 \text { soggetti adulti sani } \\
\text { ( } 9 \text { maschi e } 7 \\
\text { femmine, } 18-65 \text { anni, } \\
\text { BMI } 18-27 \mathrm{~kg} / \mathrm{m}^{2} \text { ) }\end{array}$ & $\begin{array}{l}\text { Somministrazione } \\
\text { singola }(5,20 \mathrm{mg})\end{array}$ & $\begin{array}{l}\text { - Il profilo di cortisolemia è più } \\
\text { fisiologico dopo DR-HC rispetto a HC, } \\
\text { senza differenze in Tmax tra la dose di } 5 \\
\text { e } 20 \mathrm{mg} \text {, se assunte a digiuno } \\
\text { - Il DR-HC presenta un rapido } \\
\text { assorbimento intestinale e una } \\
\text { biodisponibilità > } 90 \% \\
\text { - L'assorbimento di DR-HC dopo il pasto } \\
\text { è ritardato di circa } 60 \text { minuti rispetto } \\
\text { all'assunzione a digiuno, senza differenze } \\
\text { nel profilo di cortisolemia }\end{array}$ \\
\hline Johannsson et al. [7] & $\begin{array}{l}\text { fase } 2 \text {, aperto, } \\
\text { randomizzato, } 2 \text { bracci, } \\
2 \text { periodi }\end{array}$ & $\begin{array}{l}63 \text { pazienti con PAI } \\
(19-71 \text { anni, } 37 \text { maschi } \\
\text { e } 26 \mathrm{femmine}, \mathrm{BMI} \\
26,2 \mathrm{~kg} / \mathrm{m}^{2} ; 11 \mathrm{con} \\
\mathrm{DM}, 11 \text { con } \\
\text { ipertensione arteriosa) }\end{array}$ & $\begin{array}{l}12 \text { settimane } \\
+24 \text { settimane } \\
(20-40 \mathrm{mg})\end{array}$ & $\begin{array}{l}\text { - Il profilo di cortisolemia è più } \\
\text { fisiologico dopo DR-HC rispetto a HC } \\
\text { - Riduzione dell'esposizione al cortisolo } \\
\text { nelle } 24 \text { h del } 19,4 \% \text { dopo DR-HC } \\
\text { rispetto a HC }\end{array}$ \\
\hline Johannsson et al. [8] & $\begin{array}{l}\text { Aperto, randomizzato, } \\
4 \text { periodi }\end{array}$ & $\begin{array}{l}31 \text { soggetti adulti sani } \\
\text { (26 maschi e } 5 \\
\text { femmine, } 20-55 \text { anni, } \\
\text { BMI } 18-30 \mathrm{~kg} / \mathrm{m}^{2} \text { ) }\end{array}$ & $\begin{array}{l}\text { Somministrazione } \\
\text { singola }(5,15,20 \mathrm{mg})\end{array}$ & $\begin{array}{l}\text { - I livelli di cortisolo dopo DR-HC sono } \\
\text { più elevati rispetto ai livelli endogeni } \\
\text { nelle prime } 4 \text { ore dopo l'assunzione, } \\
\text { successivamente sono simili } \\
\text { - I parametri farmacocinetici non } \\
\text { risultano dose-dipendenti } \\
\text { - Grande variabilità inter-individuale del } \\
\text { profilo di cortisolemia. }\end{array}$ \\
\hline
\end{tabular}

insufficienza surrenalica primaria, con riduzione maggiore dell'HbA1C nel sottogruppo di 11 pazienti con diabete mellito (Tabella 2). Gli autori non hanno riscontrato differenze significative tra DR-HC e HC per quanto concerne invece il quadro lipidico, salvo una lieve diminuzione del colesteroloHDL e un modesto aumento dei trigliceridi in tutti i pazienti, ma non nel gruppo di pazienti con diabete mellito; dopo 24 settimane di trattamento si è osservata una lieve riduzione dei livelli totali di colesterolo. Il trattamento con DR-HC si è dimostrato capace di ridurre sia la pressione arteriosa sistolica che diastolica dopo 12 settimane, se confrontato con l'HC, soprattutto in pazienti con livelli più alti di pressione, ma non dopo 24 settimane di trattamento.

Nel 2015, Quinkler e collaboratori [9], in uno studio aperto, prospettico, non randomizzato, condotto in $50 \mathrm{pa}-$ zienti con insufficienza surrenalica ( 26 forme primarie di cui 15 in DR-HC, 18 forme secondarie di cui 9 in DR-HC, 6 sindromi adrenogenitali classiche in DR-HC) hanno dimostrato che il trattamento con DR-HC, per periodo variabile da 3 a 6 mesi, ha determinato una riduzione dell'indice di massa corporea (BMI), dell'HbA1c e dei livelli di colesterolo totale, mentre i livelli di colesterolo-HDL, LDL e i trigliceridi non sono cambiati in modo significativo rispetto a $\mathrm{HC}$.

Nello stesso anno, il nostro gruppo [10] ha valutato gli effetti di una terapia con DR-HC per 12 mesi alla dose di $20 \mathrm{mg}$ al giorno sui parametri antropometrici, metabolici e ormonali in 19 pazienti con malattia di Addison autoimmu- ne, precedentemente trattati con $\mathrm{HC}$ alla dose fissa di $20 \mathrm{mg}$ al giorno somministrato tre volte al giorno. Lo studio ha dimostrato che il trattamento con DR-HC determina una significativa diminuzione della circonferenza vita, dell'HbA1c, del colesterolo totale e del colesterolo LDL.

Nel 2017 Isidori e colleghi [11] in uno studio randomizzato, controllato, singolo-cieco, hanno valutato gli effetti della terapia con DR-HC in 46 pazienti con insufficienza surrenalica sia primaria che secondaria, confrontati con 43 pazienti in terapia con $\mathrm{HC}$, rispetto a un gruppo di soggetti sani comparabili per età e per sesso. Sono stati valutati gli effetti del DR-HC su parametri antropometrici, metabolici e immunitari. Gli autori hanno dimostrato che dopo 24 settimane di trattamento il DR-HC riduce il peso corporeo, il BMI, la circonferenza vita e l'HbA1C, senza modificare altri parametri metabolici, fatta eccezione per una riduzione del colesterolo HDL. Gli autori hanno inoltre dimostrato che il trattamento con DR-HC modifica il profilo delle cellule immunitarie (con riduzione dei monociti CD14+CD16-), che diventa più simile a quello dei soggetti sani di controllo, con minor suscettibilità alle infezioni.

Nel 2018 Mongioì e collaboratori [12] hanno valutato l'effetto della terapia con DR-HC in 10 pazienti con forma primaria e 9 con forma secondaria, inclusi anche 5 casi naive per DR-HC, dimostrando una riduzione dell'HbA1C solo nei pazienti con forma primaria, mentre nei pazienti con forma secondaria è stato osservato un peggioramento 
Tabella 2 Studi clinici sugli effetti del DR-HC su parametri antropometrici, metabolici e sulla qualità della vita. PAI, insufficienza surrenalica primaria; $S A I$, insufficienza surrenalica secondaria; $C A H$, sin-

\begin{tabular}{|c|c|c|c|c|}
\hline Autore & Tipo di studio & Soggetti & $\begin{array}{l}\text { durata del trt } \\
\text { (dose) }\end{array}$ & Risultati principali \\
\hline Johannsson et al. [7] & $\begin{array}{l}\text { fase } 2 \text {, aperto, } \\
\text { randomizzato, } 2 \text { bracci, } \\
2 \text { periodi }\end{array}$ & $\begin{array}{l}63 \text { pazienti con PAI } \\
\text { (19-71 anni, } 37 \text { maschi } \\
\text { e } 26 \text { femmine; } 11 \text { con } \\
\text { DM, } 11 \text { con } \\
\text { ipertensione arteriosa) }\end{array}$ & $\begin{array}{l}12 \text { settimane } \\
+24 \text { settimane } \\
(20-40 \mathrm{mg})\end{array}$ & $\begin{array}{l}\downarrow \text { peso } \\
\downarrow \text { HbA1c } \\
\downarrow \text { PAS, PAD } \\
\text { Migliora QoL }\end{array}$ \\
\hline Quinkler et al. [9] & aperto, prospettico & $\begin{array}{l}50 \text { pazienti ( } 26 \text { con } \\
\text { PAI, } 18 \text { con SAI, } 6 \text { con } \\
\text { CAH; } 19-71 \text { anni, } 37 \\
\text { maschi e } 26 \text { femmine; } \\
11 \text { con DM, } 11 \text { con } \\
\text { ipertensione arteriosa) }\end{array}$ & $\begin{array}{l}202 \text { die (mediana) } \\
85-498 \text { die (range) } \\
(15-30 \mathrm{mg})\end{array}$ & $\begin{array}{l}\downarrow \text { BMI } \\
\downarrow \text { HbA1c } \\
\downarrow \text { colesterolo totale } \\
\text { Non peggiora QoL }\end{array}$ \\
\hline Giordano et al. [10] & aperto, prospettico & $\begin{array}{l}19 \text { pazienti (PAI; } \\
27-65 \text { anni, } 4 \text { maschi e } \\
15 \text { femmine; } 4 \text { con } \\
\text { DM) }\end{array}$ & $\begin{array}{l}3-6-12 \text { mesi } \\
(20 \mathrm{mg})\end{array}$ & $\begin{array}{l}\downarrow \text { circonferenza vita } \\
\downarrow \text { HbAlc } \\
\downarrow \text { colesterolo totale, LDL, } \\
\text { Migliora QoL }\end{array}$ \\
\hline Isidori et al. [11] & $\begin{array}{l}\text { Randomizzato, } \\
\text { singolo-cieco, } \\
\text { controllato }\end{array}$ & $\begin{array}{l}89 \text { pazienti (44 PAI, } 45 \\
\text { SAI; } 46 \text { in DR-HC, } 43 \\
\text { in HC; } 27-65 \text { anni, } 4 \\
\text { maschi e } 15 \text { femmine, } \\
4 \text { con DM) }\end{array}$ & $\begin{array}{l}6 \text { mesi } \\
\left(14-18 \mathrm{mg} / \mathrm{m}^{2}\right)\end{array}$ & $\begin{array}{l}\downarrow \text { peso corporeo, BMI, circonferenza vita } \\
\downarrow \text { HbA1C }(-0,3 \%) \\
\downarrow \text { CD14+CD16-, } \uparrow \text { CD16+CD14- } \\
\downarrow \text { score di infezioni } \\
\text { Migliora QoL }\end{array}$ \\
\hline Mongioi et al. [12] & aperto, prospettico & $\begin{array}{l}19 \text { pazienti (10 PAI, } 9 \\
\text { SAI) }\end{array}$ & $\begin{array}{l}3-6-9-1-\text { mesi } \\
(28,33 \pm 6,68 \mathrm{mg})\end{array}$ & $\begin{array}{l}\downarrow \mathrm{HbA} 1 \mathrm{c} \\
\uparrow \text { sensibilità insulinica (PAI) } \\
\uparrow \text { trigliceridi (SAI) }\end{array}$ \\
\hline Ceccato et al. [13] & $\begin{array}{l}\text { Prospettico, } \\
\text { osservazionale }\end{array}$ & $\begin{array}{l}18 \text { pazienti (11 PAI, } 7 \\
\text { SAI) }\end{array}$ & $\begin{array}{l}\text { Almeno } 6 \text { mesi } \\
(25 \mathrm{mg})\end{array}$ & $\begin{array}{l}\downarrow \mathrm{HbA} 1 \mathrm{C} \\
\downarrow \text { colesterolo totale } \\
\text { Non modifica QoL }\end{array}$ \\
\hline Guarnotta et al. [14] & retrospettivo & $\begin{array}{l}49 \text { pazienti (13 PAI, } 36 \\
\text { SAI; } 24 \text { con } \\
\text { pre-diabete) }\end{array}$ & $\begin{array}{l}36 \text { mesi } \\
(20-40 \mathrm{mg})\end{array}$ & $\begin{array}{l}\downarrow \text { BMI, circonferenza vita } \\
\downarrow \text { HbA1c } \\
\uparrow \text { sensibilità/secrezione insulinica (pre-diabete) } \\
\text { Migliora QoL }\end{array}$ \\
\hline Guarnotta et al. [15] & $\begin{array}{l}\text { Basato su prove di } \\
\text { evidenza }\end{array}$ & $\begin{array}{l}53 \text { pazienti (SAI, PAI) } \\
\text { vs } 47 \text { pazienti in } \\
\mathrm{HC} / \mathrm{CA}\end{array}$ & $\begin{array}{l}48 \text { mesi } \\
(22 \pm 4,8 \mathrm{mg})\end{array}$ & $\begin{array}{l}\downarrow \text { BMI, } \downarrow \text { circonferenza vita } \\
\downarrow \text { PAD } \\
\downarrow \text { LDL-colesterolo } \\
\downarrow \text { rischio cardiovascolare }\end{array}$ \\
\hline Guarnotta et al. [16] & $\begin{array}{l}\text { Retrospettivo, } \\
\text { osservazionale }\end{array}$ & 45 pazienti (SAI) & $\begin{array}{l}12 \mathrm{mesi} \\
(20-40 \mathrm{mg})\end{array}$ & $\begin{array}{l}\downarrow \text { steatosi epatica } \\
\downarrow \text { BMI, circonferenza vita } \\
\downarrow \text { insulinemia, HOMA }\end{array}$ \\
\hline
\end{tabular}

drome adrenogenitale classica; $P A S$, pressione arteriosa sistolica; $P A D$, pressione arteriosa diastolica; $C A$, cortone acetato; $H C$, idrocortisone 
Tabella 3 Studi clinici sul profilo di sicurezza del DR-HC. PAI, insufficienza surrenalica primaria; $S A I$, insufficienza surrenalica secondaria; $C A H$, sindrome adrenogenitale classica; $P A S$, pressione arteriosa si-

\begin{tabular}{|c|c|c|c|c|}
\hline Autore & Tipo di studio & Soggetti & $\begin{array}{l}\text { durata del trt } \\
\text { (dose) }\end{array}$ & Effetti avversi \\
\hline \multirow[t]{2}{*}{ Johannsson et al. [7] } & $\begin{array}{l}\text { fase } 2 \text {, aperto, } \\
\text { randomizzato, } 2 \\
\text { bracci, } 2 \text { periodi }\end{array}$ & $\begin{array}{l}63 \text { pazienti con PAI } \\
(19-71 \text { anni, } 37 \text { maschi e } \\
26 \text { femmine; } 11 \text { con DM, } \\
11 \text { con ipertensione } \\
\text { arteriosa) }\end{array}$ & 12 settimane & $\begin{array}{l}73,4 \% \text { dei pazienti durante DR-HC } 103 \text { EAs } \\
65,6 \% \text { dei pazienti durante HC } 75 \text { EAs }\end{array}$ \\
\hline & & & $\begin{array}{l}+24 \text { settimane } \\
(20-40 \mathrm{mg})\end{array}$ & $\begin{array}{l}\text { 50,8\% dei pazienti durante DR-HC } 37 \text { EAs } \\
54,4 \% \text { dei pazienti durante HC 50 EAs } \\
5 \text { EAs severi, nessun decesso }\end{array}$ \\
\hline Nillson et al. [20] & prospettico & $\begin{array}{l}64 \text { pazienti }+16 \text { pazienti } \\
\text { (PAI) }\end{array}$ & $\begin{array}{l}24 \mathrm{mesi} \\
(20-40 \mathrm{mg})\end{array}$ & $\begin{array}{l}73,4 \% \text { dei pazienti durante } \mathrm{DR}-\mathrm{HC} \\
65,6 \% \text { dei pazienti durante } \mathrm{HC}\end{array}$ \\
\hline Giordano et al. [10] & $\begin{array}{l}\text { aperto, } \\
\text { prospettico }\end{array}$ & $\begin{array}{l}19 \text { pazienti (PAI; } 27-65 \\
\text { anni, } 4 \text { maschi e } 15 \\
\text { femmine; } 4 \text { con } \mathrm{DM})\end{array}$ & $\begin{array}{l}3-6-12 \text { mesi } \\
(20 \mathrm{mg})\end{array}$ & 7 EAs moderati nei primi 3 mesi di trattamento \\
\hline Nillson et al. [21] & $\begin{array}{l}\text { Prospettico, } \\
\text { aperto, } \\
\text { multicentrico }\end{array}$ & 71 pazienti (PAI) & $\begin{array}{l}5 \text { anni } \\
(20-40 \mathrm{mg})\end{array}$ & Buona tolleranza, nessun EAs durante i primi 5 anni \\
\hline Isidori et al. [11] & $\begin{array}{l}\text { Randomizzato, } \\
\text { singolo-cieco, } \\
\text { controllato }\end{array}$ & $\begin{array}{l}89 \text { pazienti (44 PAI, } 45 \\
\text { SAI; } 46 \text { in DR-HC, } 43 \text { in } \\
\text { HC; } 27-65 \text { anni, } 4 \text { maschi } \\
\text { e } 15 \text { femmine, } 4 \text { con DM) }\end{array}$ & $\begin{array}{l}6 \text { mesi } \\
\left(14-18 \mathrm{mg} / \mathrm{m}^{2}\right)\end{array}$ & $\begin{array}{l}\text { Nessuna differenza in frequenza e/o severità degli } \\
\text { EAs tra DR-HC e HC }\end{array}$ \\
\hline
\end{tabular}

stolica; $P A D$, pressione arteriosa diastolica; $C A$, cortone acetato; $H C$, idrocortisone; $E A s$, evento avverso 
il trattamento con DR-HC determini un miglioramento significativo dei valori di BMD a livello di colonna lombare e collo femorale, con completa normalizzazione della BMD in alcuni pazienti.

Non esistono finora studi che valutino l'effetto del DR$\mathrm{HC}$ sul rischio di frattura.

\section{Profilo di sicurezza}

In base all'analisi degli effetti avversi registrati nei diversi studi, il DR-HC presenta un profilo di sicurezza a breve e lungo termine simile a quello della terapia sostitutiva convenzionale con $\mathrm{HC}$, e la maggior parte degli eventi avversi si verifica generalmente durante i primi mesi della terapia con DR-HC (Tabella 3).

In particolare, Johannsson et al. [7] nella fase di crossover del loro studio hanno scoperto che 47 su 64 pazienti $(73,4 \%)$ hanno riportato un totale di 103 eventi avversi (EA) durante il trattamento con DR-HC, mentre 42 pazienti $(65,6 \%)$ hanno riportato $75 \mathrm{EA}$ durante la terapia convenzionale con HC. Tuttavia, nel corso della somministrazione di DR-HC solo due pazienti presentavano gravi EA. Durante il periodo di estensione di 6 mesi, 30 pazienti $(50,8 \%)$ hanno segnalato 37 EA durante i primi 3 mesi e 31 pazienti $(54,4 \%)$ hanno segnalato 50 EA durante il secondo periodo di 3 mesi. In totale, 8 gravi EA si sono verificati nella fase di crossover, 6 durante il trattamento con DR-HC e 2 durante l'HC mentre non si sono verificati decessi durante lo studio.

Nilsson e collaboratori [20] hanno condotto uno studio prospettico su 64 pazienti affetti da insufficienza surrenalica più altri 16 pazienti che sono stati aggiunti in seguito per valutare la sicurezza clinica a lungo termine del trattamento con DR-HC. Questo studio ha mostrato che entro i primi 3 mesi (fase 1) del trattamento, il tasso di EA era simile nei pazienti trattati con DR-HC rispetto ai pazienti in terapia con HC $(73,4$ vs $65,6 \%)$ e diminuito nei mesi successivi (fase 2 e 3).

Più recentemente, lo stesso gruppo ha condotto uno studio di estensione di 5 anni sulla sicurezza a lungo termine e sulla tollerabilità del trattamento con DR-HC in 71 pazienti affetti da insufficienza surrenalica [21], osservando una frequenza più alta di EA nel secondo anno di terapia, e una più bassa nell'ultimo anno. Gli autori hanno inoltre osservato che durante tutti i 5 anni di trattamento con DR-HC gli episodi di malattia intercorrente erano simili a quelli registrati durante HC.

Anche i risultati di altri studi incluso quello del nostro gruppo [10], quello di Isidori e colleghi [11] condotto in una più ampia popolazione di pazienti, non hanno mostrato EA maggiori rispetto alla terapia convenzionale, generalmente lievi e con comparsa soprattutto nei primi mesi di terapia.

\section{Conclusioni}

Il Plenadren ${ }^{\circledR}$ consente una migliore esposizione giornaliera di cortisolo nei pazienti con insufficienza surrenalica rispetto alla terapia sostitutiva convenzionale. Tale farmaco migliora alcuni parametri antropometrici e metabolici, la QoL, mentre sono ancora scarsi i dati sulla salute dell'osso. La scelta del Plenadren ${ }^{\circledR}$ rispetto alla terapia convenzionale potrebbe avvenire sulla base di quanto recentemente pubblicato dalla "Expert Opinion" della Società Italiana di Endocrinologia [22] in cui si sono cercati di individuare i migliori candidati alla nuova terapia. In particolare, i pazienti giovani con forma primaria, soprattutto se già con complicanze metaboliche o aumentato rischio cardiovascolare, parrebbero i migliori candidati alla terapia con Plenadren ${ }^{\circledR}$. Più discordanti sono le indicazioni circa il suo impiego in pazienti affetti da forma secondaria.

Funding Note Open access funding provided by Università degli Studi di Torino within the CRUI-CARE Agreement.

Conflitto di interesse Gli autori Vincenzo Cappiello, Jacopo Giannelli e Roberta Giordano dichiarano di non avere conflitti di interesse.

Consenso informato Lo studio presentato in questo articolo non ha richiesto sperimentazione umana.

Studi sugli animali Gli autori di questo articolo non hanno eseguito studi sugli animali.

Nota della casa editrice Springer Nature rimane neutrale in riguardo alle rivendicazioni giurisdizionali nelle mappe pubblicate e nelle affiliazioni istituzionali.

Open Access This article is licensed under a Creative Commons Attribution 4.0 International License, which permits use, sharing, adaptation, distribution and reproduction in any medium or format, as long as you give appropriate credit to the original author(s) and the source, provide a link to the Creative Commons licence, and indicate if changes were made. The images or other third party material in this article are included in the article's Creative Commons licence, unless indicated otherwise in a credit line to the material. If material is not included in the article's Creative Commons licence and your intended use is not permitted by statutory regulation or exceeds the permitted use, you will need to obtain permission directly from the copyright holder. To view a copy of this licence, visit http://creativecommons.org/licenses/by/4.0/.

\section{Bibliografia}

1. Husebye ES, Allolio B, Arlt W et al (2014) Consensus statement on the diagnosis, treatment and follow-up of patients with primary adrenal insufficiency. J Intern Med 275:104-115

2. Bornstein SR, Allolio B, Arlt W et al (2016) Diagnosis and treatment of primary adrenal insufficiency: an Endocrine Society clinical practice guideline. J Clin Endocrinol Metab 101:364-389

3. Bergthorsdottir R, Leonsson-Zachrisson M, Odén A et al (2006) Premature mortality in patients with Addison's disease: a population-based study. J Clin Endocrinol Metab 91:4849-4853 
4. Crown A, Lightman S (2005) Why is the management of glucocorticoid deficiency still controversial: a review of the literature. Clin Endocrinol 63:483-492

5. Giordano R, Marzotti S, Balbo M et al (2009) Metabolic and cardiovascular profile in patients with Addison's disease under conventional glucocorticoid replacement. J Endocrinol Invest 32:917-923

6. Johannsson G, Bergthorsdottir R, Nilsson AG et al (2009) Improving glucocorticoid replacement therapy using a novel modifiedrelease hydrocortisone tablet: a pharmacokinetic study. Eur J Endocrinol 161(1):119-130

7. Johannsson G, Nilsson AG, Bergthorsdottir R et al (2012) Improved cortisol exposure-time profile and outcome in patients with adrenal insufficiency: a prospective randomized trial of a novel hydrocortisone dual-release formulation. J Clin Endocrinol Metab 97:473-481

8. Johannsson G, Lennernäs H, Marelli C et al (2016) Achieving a physiological cortisol profile with once-daily dual release hydrocortisone: a pharmacokinetic study. Eur J Endocrinol 175:85-93

9. Quinkler M, Miodini Nilsen R, Zopf K et al (2015) Modifiedrelease hydrocortisone decreases BMI and $\mathrm{HbA} 1 \mathrm{c}$ in patients with primary and secondary adrenal insufficiency. Eur J Endocrinol 172(5):619-626

10. Giordano R, Guaraldi F, Marinazzo E et al (2016) Improvement of anthropometric and metabolic parameters, and quality of life following treatment with dual-release hydrocortisone in patients with Addison's disease. Endocrine 51(2):360-368

11. Isidori AM, Venneri MA, Graziadio C et al (2018) Effect of oncedaily, modified-release hydrocortisone versus standard glucocorticoid therapy on metabolism and innate immunity in patients with adrenal insufficiency (DREAM): a single-blind, randomised controlled trial. Lancet Diabetes Endocrinol 6:173-185

12. Mongiò̀ LM, Condorelli RA, La Vignera S et al (2018) Dualrelease hydrocortisone treatment: glycometabolic profile and health-related quality of life. Endocr Connect 7:211-219

13. Ceccato F, Selmin E, Sabbadin C et al (2018) Improved salivary cortisol rhythm with dual-release hydrocortisone. Endocr Connect 7:965-974
14. Guarnotta V, Ciresi A, Pillitteri G et al (2018) Improved insulin sensitivity and secretion in prediabetic patients with adrenal insufficiency on dual-release hydrocortisone treatment: a 36-month retrospective analysis. Clin Endocrinol (Oxf) 88(5):665-672

15. Guarnotta V, Di Stefano C, Santoro A et al (2019) Dualrelease hydrocortisone vs conventional glucocorticoids in adrenal insufficiency. Endocr Connect 8(7):853-862

16. Guarnotta V, Mineo MI, Radellini S et al (2019) Dual-release hydrocortisone improves hepatic steatosis in patients with secondary adrenal insufficiency: a real-life study. Ther Adv Endocrinol Metab 10:2042018819871169

17. Øksnes M, Bensing S, Hulting A et al (2012) Quality of life in European patients with Addison's disease: validity of the disease-specific questionnaire AddiQoL. J Clin Endocrinol Metab 97:568-576

18. Boesen VB, Christoffersen T, Watt T et al (2018) PlenadrEMA: effect of dual-release versus conventional hydrocortisone on fatigue, measured by ecological momentary assessments: a study protocol for an openlabel switch pilot study. BMJ Open 8(1):e019487

19. Frara S, Chiloiro S, Porcelli T et al (2018) Bone safety of dualrelease hydrocortisone in patients with hypopituitarism. Endocrine 60:528-531

20. Nilsson AG, Marelli C, Fitts D et al (2014) Prospective evaluation of long-term safety of dual-release hydrocortisone replacement administered once daily in patients with adrenal insufficiency. Eur J Endocrinol 171(3):369-377

21. Nilsson AG, Bergthorsdottir R, Burman P et al (2017) Long-term safety of once-daily, dual-release hydrocortisone in patients with adrenal insufficiency: a phase 3b, open-label, extension study. Eur J Endocrinol 176:715-725

22. Isidori AM, Arnaldi G, Boscaro M et al (2020) Towards the tailoring of glucocorticoid replacement in adrenal insufficiency: the Italian Society Endocrinology expert opinion. J Endocrinol Invest 43(5):683-696 